\title{
Development of an in-house Quantitative ELISA for the Evaluation of Different Covid-19 Vaccines in Human
}

\section{MARIEM GDOURA}

Institut Pasteur de Tunis

Fatma Ben Ghaloum

Institut Pasteur de Tunis

MERIEM BEN HAMIDA

Institut Pasteur de Tunis

WAFA CHAMSA

Institut Pasteur de Tunis

HENDA TRIKI

Institut Pasteur de Tunis

Chokri Bahloul ( $\nabla$ chokri.bahloul@pasteur.rns.tn )

Institut Pasteur de Tunis

\section{Research Article}

Keywords: Vaccine, SARS-CoV-2, ELISA, Spike, Hybrid vigor immunity

Posted Date: January 21st, 2022

DOI: https://doi.org/10.21203/rs.3.rs-1249600/v1

License: (9) (i) This work is licensed under a Creative Commons Attribution 4.0 International License.

Read Full License 


\section{Abstract}

Purpose: Reliable serological assays are needed to understand the real impact of COVID-19. In order to compare the efficiency of different COVID-19 vaccines used in the National Vaccination Program in Tunisia, we have developed a quantitative in-house ELISA.

Methods: The ELISA is based on the ectodomain of the SARS-CoV-2 Spike Baculovirus recombinant protein. We have used a panel of 145 COVID-19 RT-PCR positive serum samples and 116 pre-pandemic serum samples as a negative panel. The validation was carried out by comparison to four commercial techniques (Vidas ${ }^{\circledR}$ SARS-CoV-2 IgG anti-RBD Biomérieux ${ }^{\circledR}$, Elecsys ${ }^{\circledR}$ Anti-Nucleocapsid of SARS-CoV-2 Roche ${ }^{\circledR}$, cPass ${ }^{\circledR}$ GenScript ${ }^{\circledR}$ and the quantitative Elecsys ${ }^{\circledR}$ Anti-RBD of SARS-CoV-2, Roche $\left.{ }^{\circledR}\right)$. For the evaluation of the National Vaccination campaign, we have included 115 recipients of one of the different used vaccines.

Results: The qualitative performances of the developed ELISA gave $96 \%$ sensitivity, $97.5 \%$ specificity and 0.968 accuracy. For the evaluation of the different brand of vaccines in recipients not previously infected with SARS-CoV-2, it seems that mRNA vaccine of Pfizer/BioNTech has shown a higher efficacy compared to inactivated virus vaccines. COVID-19 convalescent individuals have generated poor antibody responses. Nevertheless, when they are vaccinated with any brand of the COVID-19 vaccines, many of them mounted an exponential increase of the induced immune responses, qualified as a "hybrid vigor immunity".

Conclusion: Our developed in-house ELISA seems to be very efficient in evaluating the effectiveness of anti-COVID-19 vaccination. Platforms based on mRNA vaccine are better performing than that based on inactivated virus.

\section{Introduction}

The ongoing global pandemic of coronavirus disease 2019 (COVID-19) is caused by the Severe Acute Respiratory Syndrome Coronavirus 2 (SARS-CoV-2), identified for the first time in December 2019 in Wuhan, China. The disease was declared by the World Health Organization (WHO) as a pandemic on the 11th of March 2020 [1]. Up to 23 December 2021, more than 276 million cumulative cases and 5.3 million cumulative deaths have been reported [2]. The high burden of the disease triggered a race towards the development and distribution of effective vaccines, in order to slow the viral transmission, lower the disease severity and reduce the mortality. While typical vaccine development can take up to 10-15 years, COVID-19 vaccines needed less than a year after the identification of COVID-19 [3]. To reach such challenge, clinical development, manufacturing scale-up and distribution are occurring in parallel for all the COVID-19 vaccines. Until 21 December 2021, 137 vaccines are under clinical trials, with 10 in Phase 4 after being approved for emergency use in humans [4]. Different platforms of approved vaccines are currently largely administered to humans all over the world, among which mRNA vaccines (PfizerBioNTech, Moderna); recombinant adenovirus vectored vaccines (AstraZeneca, Cansino, Gamaleya, 
Johnson Pharm) and inactivated vaccines (Sinopharm, Sinovac). Phase 4 clinical trial, also called postmarketing surveillance trial, studies the side effects caused over time that were not seen in earlier trials by a new vaccine after it has been approved and is on the market. It may also study how well the vaccine works over a long period.

Although the introduction of Covid-19 vaccines contributed to decrease the burden of the disease in many parts of the world, new cases have increased significantly in many countries in both vaccinated and nonvaccinated populations due to the emergence of new variants [5]. In order to prevent new ongoing SARSCoV-2 variants, massive vaccination of people around the world should be accomplished as early as possible. In addition, it seems that mRNA vaccines including Pfizer/BioNTech and Moderna have shown higher efficacy and protection against COVID-19 infection [5].

Vaccines against COVID-19 are not reaching many people in developing countries. Less than $1 \%$ of people in low-income countries are fully vaccinated, and just $10 \%$ are in lower-middle-income countries, compared with more than half in high-income countries [6]. In Tunisia, from 3 January 2020 to 23 December 2021, there have been 721,797 confirmed cases of COVID-19 and 25,491 deaths, reported to WHO. As of 23 December 2021, more than 12.6 million vaccine doses have been administered [7]. Up to 20 December 2021, fully vaccinated people in Tunisia were 46.7\% [8]. In Tunisia, all the platforms of COVID-19 vaccines have been used (Pfizer/Biontech, Moderna, Spoutnik, AstraZeneca, Johnson \& Johnson, Sinovac and Sinopharm).

Recent studies indicate that binding and neutralizing SARS-CoV-2 antibodies elicited by natural infection or vaccination persist for more than 6 months although their concentration decreases over time [9]. The passive transfer of neutralizing antibodies and protection are correlated in non-human primates [10]. While such a link has not yet been defined in humans, individuals with high neutralizing antibody titers could well be better protected against SARS-CoV-2. It was reported that neutralizing antibodies are highly correlated with protection due to their ability to block the viruses from entering the host cells [11]. A recent investigation in France reported that ELISA concentrations between 13 and 141 BAU (Binding Antibody Units)/ml provided only $12.4 \%$ protection against SARS-CoV-2; Concentrations between 141 and 1700 $\mathrm{BAU} / \mathrm{ml}$ provided $89.3 \%$ protection; And concentrations of $1700 \mathrm{BAU} / \mathrm{ml}$ and above provided full protection [12]. Therefore, reliable serology, with highly specific and sensitive performances are urgently needed in order to evaluate the efficiency of the administered COVID-19 vaccines.

In our investigation, we have set up and validated a quantitative ELISA technique using a Bacculovirus recombinant full length of the SARS-CoV-2 Spike Glycoprotein. This ELISA technique allowed us to assess and compare the efficiency of the different COVID-19 vaccines used in the National Vaccination Program in Tunisia.

\section{Material \& Methods}

\section{Patient sampling and ethical issues}


The sampling was carried out in the Laboratory of Virology of the "Institut Pasteur de Tunis", Tunisia. For serotheque constitution, an ethical approval was obtained from the Institutional Review Board of the "Institut Pasteur de Tunis" (Reference: 2020/27/I/LR16IPT). All the samples were collected from patients after being issued a written consent. The selection of samples followed the guidelines of the French "Centre National de Reference des Virus des Infections Respiratoires" published on December 4th 2020, i.e. the evaluation needs at least 50 true positive sera and at least 50 true negative sera. In order to validate our in house ELISA, we have used a positive and a negative, panels. The positive panel was composed of 145 unique, non-duplicated serum samples obtained from COVID-19 confirmed patients based on a positive RT-PCR on nasopharyngeal swab. Samples were collected from the first day (D0) until 109 day (D109) after molecular confirmation. Serum samples included 20 sera collected from D1 to D14, 27 sera collected from D15 to D21, 17 sera collected from D22 to D30, 23 sera collected from D31 to D60 and 17 sera collected from D61 to D 109. The negative panel was composed of 116 pre-pandemic sera collected before December 2019. For the evaluation of the National Vaccination campaign against COVID-19 in Tunisia, we have recruited 115 recipient of one of the different used vaccines.

\section{Recombinant protein}

For plate coating of the in-house ELISA we have used the SARS-CoV-2 (2019-nCoV) Spike S1+S2 ECD-His Recombinant Protein Baculovirus-Insect Cells (Sinobiological, China). It consists of 1209 amino acids and predicts a molecular mass of $134.36 \mathrm{kDa}$.

\section{COVID-19 vaccines}

For the National Vaccination Program against the COVID-19 in Tunisia we have used the following vaccines:

- The mRNA BNT162b2 (Pfizer, Inc., USA and BioNTec, Germany) administered in two shots, 21 days apart into the muscle of the upper arm.

- The mRNA-1273 (Moderna, USA) administered in two shots, 28 days apart into the muscle of the upper arm.

- The Adenovirus Vector JNJ-78436735 (Janssen Pharmaceuticals Companies of Johnson \& Johnson, USA) administered in one shot into the muscle of the upper arm.

- The Adenovirus Vector Vaxzevria (previously COVID-19 Vaccine AstraZeneca, UK) is given as two injections, usually into the muscle of the upper arm. The second dose should be given between 4 and 12 weeks after the first dose.

- The Adenovirus Vector Sputnik V (Gamaleya Research Institute of Epidemiology and Microbiology, Russia) administered in two intramuscular shots, by using one type of adenovirus, Ad26, for the first dose, and another, Ad5, for the second.

- The inactivated viruses Coronavax (Sinovac, China) and Sinopharm (Sinopharm, China) used as two doses intramuscularly. WHO recommends an interval of 3-4 weeks between the first and the second dose. 


\section{Enzyme-linked immunosorbent assays}

To set up our in-house quantitative ELISA for COVID-19 serology standard techniques were applied. Briefly, 96 well microtiter plates (Nunk) were coated with the SARS-CoV-2 Spike Baculovirus recombinant protein at the concentration of $50 \mathrm{ng} /$ well in $50 \mu \mathrm{l}$ of $0.1 \mathrm{M}$ carbonate bi-carbonate buffer $(\mathrm{pH}$ 9.6). The plates are incubated overnight at $4^{\circ} \mathrm{C}$ then saturated with $5 \%$ of bovine serum albumin (BSA) and $0.3 \%$ sucrose $\left(50 \mu \mathrm{l} /\right.$ well) in carbonate bicarbonate buffer for 30 minutes at $37^{\circ} \mathrm{C}$. After washing with PBS containing $0.05 \%$ of Tween 20 (PBS/T), $50 \mu \mathrm{l}$ of the serum to be assayed is diluted to $1 / 50$ in PBS/T containing $0.5 \%$ of BSA, in addition to an international standard after multiple dilutions are deposited in the corresponding wells and the plate is incubated at $37^{\circ} \mathrm{C}$ for one hour. Then, the plate is washed with $\mathrm{PBS} / \mathrm{T}$ and incubated in presence of peroxidase conjugate (anti-Human total IgG, Sigma) at $37^{\circ} \mathrm{C}$ for one hour. After washing, we distribute $50 \mu \mathrm{l} /$ well of TMB (Sigma) and incubate the plate in dark, 30 minutes at room temperature. The reaction is stopped by $50 \mu \mathrm{l}$ of $\mathrm{HCl}(10 \%)$ and read at $450 \mathrm{~nm}$ filter spectrophotometer.

The calibration of our in-house ELISA was carried out against the NIBSC 136/20 (National Institute for Biological Standards and Control, UK), which is the WHO international standard. Its titers are $1300 \mathrm{IU} / \mathrm{ml}$ in serum virus neutralization and $476 \mathrm{BAU} / \mathrm{ml}$ in anti-Spike IgG ELISA.

For validation of our in-house ELISA we have compared our results to four commercial serological tests All of them are authorized for the in vitro diagnosis and have been approved by the food and drug administration by having an emergency use authorization (FDA-EUA). We have tested the same samples by both the commercial assay and the in-house ELISA following exactly the manufacturer's procedures.

The first is Vidas ${ }^{\circledR}$ SARS-COV-2 IgG anti-RBD Biomérieux ${ }^{\circledR}$ FDA-EUA, which is an automated assay using the ELFA (Enzyme Linked Fluorescent Assay) technique intended for qualitative detection of IgG antibodies to SARS-CoV-2 in human serum or plasma. The second is Elecsys ${ }^{\circledR}$ Anti-Nucleocapsid of SARS-CoV-2 Roche ${ }^{\circledR}$ FDA-EUA which is an automated assay based on the ECLIA technique (Electrochimiluminescence) for the qualitative detection of total anti-N antibodies (mainly lgG) to SARSCoV-2 in human serum and plasma. The third is an ELISA surrogate serum virus neutralization test (cPass ${ }^{\circledR}$ GenScript ${ }^{\circledR}$ FDA-EUA); The cPass ${ }^{\circledR}$ technology is an ELISA and allows a rapid detection of total virus neutralizing antibodies in a sample by mimicking the interaction between the virus and the host cell receptor ACE2. The fourth is Elecsys ${ }^{\circledR}$ Anti-RBD of SARS-CoV-2, Roche ${ }^{\circledR}$ (quantitative detection of total anti-RBD, FDA-EUA). This test is calibrated against the WHO international standard.

\section{Statistical analysis}

MedCalc ${ }^{\circledR}$ V18.2.1 was used for statistical analysis: comparison of two means by t-test, correlation between two quantitative variables by Pearson Correlation, construction of ROC (Receiver Operating Characteristic) curves, determining the analytical performances: sensitivity, specificity, area under the curve (AUC). The p-values lower than 0.05 are significant at the $95 \%$ confidence interval and higher than that are not significant. We have used the USA-Food and Drug Administration (FDA) calculator available 
on its website in order to calculate the positive and negative predictive values, on the basis of a prevalence arbitrary fixed by this calculator at $5 \%$.

\section{Results}

\section{Validation of an in-house ELISA}

We have estimated the qualitative (seropositive or seronegative) performances of our in-house ELISA among several COVID-19 RT-PCR positive samples. ROC analysis (Figure 1) gave us very satisfactory performances, the overall sensitivity was $96 \% \mathrm{Cl} 95 \%$ [91.5\%-98.5\%] and the sensitivity after D14 is $95.9 \%$ CI95\% [89.8\%-98.9\%] (93 true positive out of 97 patients sampled after D14). For specificity calculation, we have used 116 pre-pandemic sera collected in 2017 as true negative sera. Hence, the specificity of our in-house ELISA was $97.5 \% \mathrm{Cl} 95 \%$ [92.8\%-99.5\%]. The accuracy of the test was very high $0.968 \mathrm{Cl} 195 \%$ [0.939-0.985] and the positive and negative predictive values were $66.7 \% \mathrm{Cl} 5 \%$ [39.6\%-85.9\%] and 99.8\% CI95\% [99.4\%-99.9\%], respectively. The comparison of our qualitative results to those of the Vidas $\AA$ antiRBD and Elecsys ${ }^{\circledR}$ anti-N showed better performances than the first (AUC 0,841 Cl95\%[0,727-0,883], pvalue $<0.05)$ but similar performances with the second $(0,885 \mathrm{Cl} 95 \%[0,808-0,938]$, p-value $>0.05)$ (Figure 2). Therefore, we can consider our developed in-house ELISA is at least as good as the commercial techniques.

Then, we have compared the quantitative results of our in-house ELISA to those of the commercial cPass ${ }^{\circledR}$ GenScript ${ }^{\circledR}$ FDA-EUA. Correlation between anti-S titers and percentage of inhibition by the surrogate sero-netralization ELISA showed a strong correlation ( $r=0.5 \mathrm{Cl} 95 \%$ [0.236-0.696], $p$-value $<0.05$ ) (Figure 3).

According to the $\mathrm{cPass}{ }^{\circledR}$ a cut-off of $30 \%$ is recommended by the manufacturer as an indicator of the presence of virus neutralizing antibodies. In extrapolation to our in-house test, this corresponds to serums with titers of $59 \mathrm{BAU} / \mathrm{ml}$.

\section{Evaluation of the humoral immune responses induced by different COVID-19 vaccines.}

The in-house validated ELISA for COVID-19 serology seems to be efficient in assessing the immune responses induced by the different vaccines used in the National Vaccination Program of Tunisia. We have analyzed 115 recipient of a COVID-19 vaccine and analyzed their immune responses by the quantitative in-house ELISA. Since most of the vaccine platforms are based only on the SARS-CoV-2 Spike and since a history of COVID-19 in vaccine recipients cannot be always confirmed, we have analyzed all the serum samples for anti-N antibodies. Hence, we have discriminated vaccine recipients of either BNT162b2, mRNA-1273, JNJ-78436735, Vaxzevria, or Sputnik V with Elecsys ${ }^{\circledR}$ anti-N. When it is positive, we consider the vaccine recipient previously SARS-CoV-2 infected and when it is negative as never infected by the virus. For vaccine recipients of inactivated viruses (Coronavax and Sinopharm), we have shown that the vaccine induced anti-N antibodies are almost undetectable. Therefore, in our 
analysis when we have high anti-N Elecsys ${ }^{\circledR}$ titres of Coronavax and Sinopharm, they are considered previously infected by the SARS-CoV-2.

All the results are compiled in Table 1, and they are grouped by vaccine brand and sub grouped according to a previous SARS-CoV-2 infection and the number of received shots. In addition, the titers were classified in different intervals and correspond to the percentages of each category of vaccine recipients in each interval. Based on Dimeglio et al. report (12), we have chosen the following intervals of antibody titers. Lower than $10 \mathrm{BAU} / \mathrm{ml}$ as seronegative; Between 10 and $50 \mathrm{BAU} / \mathrm{ml}$ as a poor immune response; Between 50 and $200 \mathrm{BAU} / \mathrm{ml}$ as borderline protective immune response; Between 200 and $400 \mathrm{BAU} / \mathrm{ml}$ as satisfactory immune response; Between 400 and 2,500 BAU/ml as high immune response; Between 2,500 and $10,000 \mathrm{BAU} / \mathrm{ml}$ as very high immune response; And higher than 10,000 as hyperimmune response.

In convalescent COVID-19 Patients sampled before the beginning of the vaccination campaign, only $1 \%$ mounted very or high immune responses in each group. They represent $11 \%$ with satisfactory immune responses. The remaining have either induced borderline protective immune responses (42.6\%), or a poor response (35\%) and even seronegative (10\%). Mean antibody titer of all the convalescent patients was 118.23 BAU/ml.

For recipients of Pfizer/Biontech, when only one shot is administered and when their anti-N Elecsys ${ }^{\circledR}$ test were negative, the induced immune responses were either satisfactory or borderline in $16.7 \%$ and $25 \%$, respectively. The remaining were either seronegative (16.7\%) or mounted a poor immune response $(41.7 \%)$. Overall, the mean antibody titer was $126 \mathrm{BAU} / \mathrm{ml}$, roughly the same as in convalescent patients. When the second shot was administered, there is still $36.4 \%$ of them with borderline protective responses. The remaining of them have reached at least satisfactory immune responses with $45.5 \%$ as high responder. The mean antibody titer was $532 \mathrm{BAU} / \mathrm{ml}$, largely higher than convalescent patients ( $p$-value 0.0004). In the Tunisian COVID-19 vaccination program, whenever a person is previously confirmed infected, they only receive one shot of vaccination. In this category of vaccine recipients, we have also confirmed an eventual previous infection by an anti-N Elecsys ${ }^{\circledR}$ positive test. Hence, in this category of Pfizer/Biontech vaccine recipients, hypeimmunized (16.1\%) and inducer of very high (61.3\%) or high (13\%) immune responses were detected. Only $6.5 \%$ and $3 \%$ of them induced rather satisfactory or poor immune responses, respectively. The mean antibody titer was $7,962 \mathrm{BAU} / \mathrm{ml}$, very largely higher than convalescent patients ( $p$-value 0,000000 ) and to two doses of Pfizer/Biontech vaccine COVID-19 negative recipients ( $p$-value 0.043 ). When people received two shots of Pfizer/Biontech vaccine, normally they should have not been previously infected by the SARS-CoV-2. However, since sometimes there are lacks of transparency from vaccine recipients, or due to the occurrence of a mild form of the disease not easy to diagnose, they can be administered two shots of Pfizer/Biontech vaccine. Nevertheless, with the anti-N Elecsys ${ }^{\circledR}$ positive test, we have been able to identify them. Although, only six of them are here represented, half of them have mounted rather borderline protective immune responses. The remaining have mounted either high, or very high or hyperimmune responses (one recipient in each category). Their mean antibody titer was $3,064 \mathrm{BAU} / \mathrm{ml}$. 
Recipients of Moderna mRNA COVID-19 vaccine are only represented by 5 individuals, all of them previously infected by the SARS-CoV-2, as confirmed by a positive anti-N test and their mean antibody titer was 1,707 BAU/ml. They have received only a single shot of vaccine and they have mounted either high (four recipients) or very high (one-recipient) immune responses.

For individuals that have received inactivated virus vaccines we have plotted the results of both brands (Sinovac and Sinopharm). When receiving only one shot of the vaccine, most of them (66.7\%) mounted borderline immune responses. The remaining either mounted a satisfactory immune response or were seronegative, at rates of $16.7 \%$ in each category. Their mean antibody titer was $132 \mathrm{BAU} / \mathrm{ml}$ very close to that of convalescent patients. When two doses of inactivated virus vaccines were administered, there is still $16.7 \%$ of them seronegative. The remaining either mounted poor or borderline immune responses (41.7\%, each group). Their mean antibody titer was $49 \mathrm{BAU} / \mathrm{ml}$, non-statistically different to that of convalescent patients ( $p$-value 0.439) and to one shot recipient ( $p$-value 0.06). In presumably infected persons, based on a positive anti-N serology, inactivated virus vaccine recipients induced almost the same level of immune responses, whether they received one, two or three shots. Hence, more than $40 \%$ of them induced high immune responses and around $40 \%$ satisfactory antibody titers. Their pooled mean antibody titer was $458 \mathrm{BAU} / \mathrm{ml}$, higher to convalescent patients ( $\mathrm{p}$-value 0.0004) and lower to Pfizer/Biontech COVID-19 positive recipients after one shot ( $p$-value 0.027) or two shots ( $p$-value 0.05).

For vaccines based on recombinant adenoviruses, only few recipients could be recruited in this investigation. After vaccination with one or two shots of AstraZeneca vaccine, SARS-CoV-2 infected individuals mounted at least high level of antibody titers. With Johnson and Johnson vaccine only three recipients were recruited, two of them did not seroconvert and the third only mounted a borderline protective response. Finally, with Sputnik V vaccine, non-infected recipients induced a rather borderline response in at least $60 \%$ of them. Out of two infected individuals and vaccinated with Sputnik V, there is one with a very high level of induced immune response. The second recipient induced a satisfactory immune response.

\section{Discussion}

The emergence of viral threats or epidemic is more and more attributable to zoonotic pathogens. Nearly $75 \%$ of the newly emerging diseases were linked to an animal major origin [13]. Although zoonosis have been thoroughly investigated, they have undergone dramatic changes, due to climate change, human impact on nature, transport of people animals and food, etc. Consequently, the relationship between man and animal has changed, facilitating the emergence or re-emergence of new zoonosis. Epidemics may threaten all countries, with health, social and economic consequences, as well as a strong media impact. These threats have major consequences on public opinion and not only health professionals must respond very quickly, but also science and researchers should adapt consequently. COVID-19 is a good illustration of this dilemma. The disease was declared by the WHO as a pandemic on the 11th of March 2020 [1] with very high human and economic consequences. Up to 9 November 2021, more than 5 million cumulative deaths have been reported [2]. The estimated drop in economic growth is 4.5 percent, only for 
2020. The global GDP in 2019 is around 87.55 trillion U.S. dollars, which means an economic output loss of almost 3.94 trillion U.S. dollars [14]. Hence, fighting against the disease is of great importance. For that we need first, to set up platforms for diagnosis and survey and second, apply adequate control measures including the development of efficacious vaccines, whenever that is possible.

Reliable serological assays are needed to understand the real impact of COVID-19 through seroepidemiological studies, as most of the cases are asymptomatic and those with mild symptoms are mostly undetected [15]. Testing specific antibody response to SARS-CoV-2 infection may help establishing diagnosis or predicting the clinical outcomes $[16,17]$. Furthermore, serology may predict the level of protection against reinfection or infection after vaccination. Establishing serum virus neutralization techniques for assaying the induced immune responses remain the gold standard. However, neutralization assays are time-consuming and expensive to perform and requires biosafety level 3 laboratory conditions due to the use of pathogenic virus. They are usually not feasible for routine laboratories especially in developing countries. Therefore, specific antibody titers are an alternative to be used as "correlate" or "surrogate" of protection if they correlate with the result of neutralization test. We have developed an in-house ELISA based on the full length of spike ectodomain of SARS-CoV-2 produced on Bacculovirus system. We were able to obtain Sensitivity higher than $95 \%$ and a Specificity of $97.5 \%$, very close performance to what was reported [18]. The French recommendations for IgG or total Ig assays at 14 days post infection must be greater than $90 \%$ and $98 \%$ for sensitivity and specificity, respectively [19]. Hence, our in-house ELISA is valid according to these recommendations. In addition, we have compared the performances of our technique to commercially available techniques (Elecsys and VIDAS) and they were at least as good as they were. It was reported that The Elecsys ${ }^{\circledR}$ and the VIDAS ${ }^{\circledR}$ methods demonstrated high sensitivities with no false positive results [20]. Furthermore, our in-house developed ELISA showed a good correlation with the quantitative automated anti-RBD test Elecsys $®$. Both were calibrated against the NIBSC 136/20 standard according to the WHO recommendations. Correlations of serology tests with neutralization tests were also demonstrated for many commercial techniques [21, 22]. Therefore, our developed in-house ELISA seems to be very efficient in evaluating the effectiveness of antiCOVID-19 vaccination.

It is largely accepted that vaccines remain the most effective tool for preventing infectious diseases and improving global health [23]. However, difficulties facing vaccinologists include predicting the type and timing of the next pandemic; developing vaccines to combat rapidly changing pathogens; and establishing rapid-response strategies to control emerging and reemerging infectious diseases. Against COVID-19, developed vaccines have shown acceptable safety records and could effectively reduce the death, severe cases, symptomatic cases, and infections resulting from SARS-CoV-2 across the world [24]. In the context of global pandemic and the continuous emergence of SARS-CoV-2 variants, accelerating vaccination and improving vaccination coverage is still the most important target.

Tunisia started its National Vaccination Program against COVID-19 on the 13th of March 2021. All the WHO authorized vaccine platforms have been used in this program. A proper assessment of the efficiency of the used vaccines has been addressed by serology surveys using our developed in-house 
ELISA. As far as when humoral immune responses are considered, and when only vaccine recipients were not previously infected with SARS-CoV-2, it seems that mRNA vaccine of Pfizer/BioNTech have shown a higher efficacy compared to inactivated virus vaccines, similar to what has been reported elsewhere [5]. For the other surveyed vaccine platforms, the enrolled COVID-19 naif recipients are rather low and robust interpretations cannot be driven. In a systematic review and meta-analysis of 58 studies, the pooled vaccine effectiveness was $85 \%(80-91 \%)$ for the prevention of Alpha variant of SARS-CoV-2 infections, $75 \%(71-79 \%)$ for the Beta variant, $54 \%$ (35-74\%) for the Gamma variant, and $74 \%$ (62-85\%) for the Delta variant [24]. It seems undoubtable that all the current COVID-19 vaccines have a drop of effectiveness against the emerging variants. Even though, Pfizer/Biontech full-vaccinated individuals induced at least borderline immune responses, but with the drop of the titers over time the level of induced antibodies [25] and with the emergence of the different variants, it is recommended to administer a third shot of the vaccine [26]. The situation is still worse with the inactivated virus vaccines, since they induce lower and quickly declining humoral immune responses [27].

We have shown that COVID-19 convalescent individuals have only generated poor or borderline protective antibodies in almost $90 \%$ of them. Nevertheless, when they are vaccinated with any brand of COVID-19 vaccines, they have mounted an exponential increase of the induced immune responses. It was reported that when previously infected individuals are vaccinated an impressive synergy occurs, giving what the authors qualified as a "hybrid vigor immunity" $[28,29]$. When natural immunity to SARS-CoV-2 is combined with vaccine, it generated a larger than expected immune responses. Hence, we were able to detect very high immune and even hyperimmune responses (titers higher than 10,000BAU/ml) especially after vaccination with Pfizer/Biontech mRNA. It was reported that differences between the memory B cells triggered by infection and those triggered by vaccination could explain the potent hybrid immunity $[30,31]$. Nevertheless, vaccination of convalescent individuals did not give systematically high immune responses. In our investigation, $50 \%$ of anti-N positive individuals vaccinated with two shots of BNT162b2, only developed borderline protective immune responses. Nevertheless, we should mention that in the National Vaccination Program of Tunisia normally COVID-19 patients receive only one shot of the vaccine. Since they have received two shots that means they have previously developed a milder or a silent form of the disease and they were not aware of that. Multiple factors contribute to the degree of immune response mounted following infection. Both binding and neutralizing antibody titers rise faster and reach a higher peak in persons with more severe COVID-19 [32, 33]. Hence, we can speculate that asymptomatic or paucisymptomatic COVID-19 patients do not develop a potent hybrid immunity after being vaccinated.

\section{Declarations}

\section{Acknowledgments:}

We would like to acknowledge the valuable help of Dr Habib Halouani and Dr Donia Sahli for valuable help in sample collection and for Mrs Nahed Hogga for technical assistance. 


\section{Competing Interests:}

The authors declare no competing interests.

\section{Availability of Data and Materials:}

The datasets used and/or analysed during the current study available from the corresponding author on reasonable request.

\section{References}

1. WHO Director-General's opening remarks at the media briefing on COVID19 -March 2020.

https://www.who.int/director-general/speeches/detail/who-director-general-s-opening-remarks-at-themedia-briefing-on-covid-19-11-march-2020.

2. WHO: WHO Coronavirus (COVID-19) Dashboard. https://covid19.who.int/

3. Burgos RM, Badowski ME, Drwiega E, Ghassemi S, Griffith N, Herald F, Johnson M, Smith RO, Michienzi SM. The race to a COVID-19 vaccine: opportunities and challenges in development and distribution. Drugs Context. 2021; https://doi.org/10.7573/dic.2020-12-2.

4. WHO: COVID-19 vaccine tracker and landscape. https://www.who.int/publications/m/item/draftlandscape-of-covid-19-candidate-vaccines.

5. Ghasemiyeh P, Mohammadi-Samani S, Firouzabadi N, Dehshahri A, Vazin A. A focused review on technologies, mechanisms, safety, and efficacy of available COVID-19 vaccines. Int Immunopharmacol. 2021; https://doi.org/10.1016/j.intimp.2021.108162.

6. Amy Maxmen. The fight to manufacture COVID vaccines in lower-income countries. Nature. 2021; https://doi.org/10.1038/d41586-021-02383-z.

7. WHO: https://covid19.who.int/region/emro/country/tn

8. Evax: https://www.evax.tn/

9. Doria-Rose N, Suthar MS, Makowski M, O'Connell S, McDermott AB, Flach B. Antibody persistence through 6 months after the second dose of mRNA-1273 vaccine for COVID-19. N Engl J Med. 2021; https://doi.org/10.1056/NEJMc2103916.

10. McMahan K, Yu J, Mercado NB, Loos C, Tostanoski LH, Chandrashekar A. Correlates of protection against SARS-CoV-2 in rhesus macaques. Nature. 2021; https://doi.org/10.1038/s41586-020-030416.

11. Douxfils J, Gillot C, Mullier F, Favresse J. Post-SARS-CoV-2 vaccination specific antibody decrease thresholds for determining seroprevalence and seroneutralization differ. J Infect. 2021; https://doi.org/10.1016/j.jinf.2021.08.023.

12. Dimeglio $\mathrm{C}$, Herin F, Martin-Blondel G, Miedougé $M$, Izopet $J$. Antibody titers and protection against a SARS-CoV-2 infection. J Infect. 2021; https://doi.org/10.1016/j.jinf.2021.09.013. 
13. Jones KE, Patel NG, Levy MA, Storeygard A, Balk D, Gittleman JL, Daszak P. Global trends in emerging infectious diseases. Nature. 2008; https://doi.org/10.1038/nature06536.

14. Szmigiera M. Impact of the coronavirus pandemic on the global economy - Statistics \& Facts. https://www.statista.com/topics/6139/covid-19-impact-on-the-globaleconomy/\#dossierKeyfigures.

15. Knezevic I, Mattiuzzo G, Page M, Minor P, Griffiths E, Nuebling M, Moorthy V. WHO International Standard for evaluation of the antibody response to COVID-19 vaccines: call for urgent action by the scientific community. The Lancet Microbe. 2021; https://doi.org/10.1016/S2666-5247(21)00266-4.

16. Carter LJ, Garner LV, Smoot JW, Li Y, Zhou Q, Saveson CJ, Sasso JM, Gregg AC, Soares DJ, Beskid TR, Jervey SR, Liu C. Assay techniques and test development for COVID-19 diagnosis. ACS Cent Sci. 2020; https://doi.org/10.1021/acscentsci.0c00501. 5.

17. Zhao J, Yuan Q, Wang H, Liu W, Liao X, Su Y, Wang X, Yuan J, Li T, Li J, Qian S, Hong C, Wang F, Liu Y, Wang Z, He Q, Li Z, He B, Zhang T, Fu Y, Ge S, Liu L, Zhang J, Xia N, Zhang Z. Antibody responses to SARS-CoV-2 in patients of novel coronavirus disease 2019. Clin Infect Dis. 2020; https://doi.org/10.1093/cid/ciaa344.

18. Alandijany TA, El-Kafrawy SA, Tolah AM, Sohrab SS, Faizo AA, Hassan AM, Alsubhi TL, Othman NA, Azhar El. Development and Optimization of In-house ELISA for Detection of Human IgG Antibody to SARS-CoV-2 Full Length Spike Protein. Pathogens. 2020;

https://doi.org/10.3390/pathogens9100803.

19. JORF $n^{\circ} 0293$ du 4 décembre 2020 Texte $n^{\circ} 55$ : https://www.legifrance.gouv.fr/eli/arrete/2020/12/3/SSAZ2033724A/jo/texte

20. Wolffa F Dahmab H, Dutermea C, Van den Wijngaertb S, Vandenbergcd O, Cottona F, Montesinos I. Monitoring antibody response following SARS-CoV-2 infection: diagnostic efficiency of 4 automated immunoassays. Diagn Microbiol Infect Dis. 2020; https://doi.org/10.1016/j.diagmicrobio.2020.115140.

21. Szabó Z, Szabó T, Bodó K, Kemenesi G, Földes F, Kristóf K, Barabás E, Vásárhelyi B, Prohászka Z, Fodor E, Jakab F, Berk T, Laczag Z. Comparison of virus neutralization activity and results of 10 different anti-SARS-CoV-2 serological tests in COVID-19 recovered plasma donors. Pract Lab Med. 2021; https://doi.org/10.1016/j.plabm.2021.e00222.

22. Grzelak L, Temmam S, Planchais C, Demeret C, Tondeur L et al. A comparison of four serological assays for detecting anti-SARS-CoV-2 antibodies in human serum samples from different populations. Sci Transl Med. 2020; https://doi.org/10.1126/scitranslmed.abc3103.

23. Julie L, Gerberding MD, Barton F, Haynes MD. Vaccine Innovations Past and Future. N Engl J Med. 2021; https://doi.org/10.1056/NEJMp2029466.

24. Liu Q, Qin C, Liu M, Liu J. Effectiveness and safety of SARS-CoV-2 vaccine in real-world studies: a systematic review and meta-analysis. Infect Dis Poverty. 2021; https://doi.org/10.1186/s40249-02100915-3. 
25. Mahase E. Covid-19: Antibody levels fall after second Pfizer dose, but protection against severe disease remains, studies indicate. BMJ. 2021; https://doi.org/10.1136/bmj.n2481.

26. Nanduri S, Pilishvili T, Derado G, Soe MM, Dollard P, Wu H, Li Q, Bagchi S et al. Effectiveness of PfizerBioNTech and Moderna Vaccines in Preventing SARS-CoV-2 Infection Among Nursing Home Residents Before and During Widespread Circulation of the SARS-CoV-2 B.1.617.2 (Delta) Variant National Healthcare Safety Network, March 1-August 1, 2021. CDC Weekly / August 27, 2021 / 70 (34); 1163-1166. https://www.cdc.gov/mmwr/volumes/70/wr/mm7034e3.htm.

27. Mallapaty S. China's COVID vaccines have been crucial - now immunity is waning. Nature. 2021; https://doi.org/10.1038/d41586-021-02796-w.

28. Crotty C. Hybrid immunity. Science. 2021; https://doi.org/10.1126/science.abj2258.

29. Callaway E. COVID super-immunity: one of the pandemic's great puzzles. Nature. 2021; https://doi.org/10.1038/d41586-021-02795-x.

30. Wang Z, Muecksch F, Schaefer-Babajew Z, Finkin S, Viant S, Gaebler C et al. Naturally enhanced neutralizing breadth against SARS-CoV-2 one year after infection. Nature. 2021; https://doi.org/10.1038/s41586-021-03696-9.

31. Cho A, Muecksch M, [...] Nussenzweig MC. Anti-SARS-CoV-2 receptor-binding domain antibody evolution after mRNA vaccination. Nature. 2021; https://doi.org/10.1038/s41586-021-04060-7.

32. Gudbjartsson DF, et al. Humoral Immune Response to SARS-CoV-2 in Iceland. N Engl J Med. 2020; https://doi.org/10.1056/NEJMoa2026116.

33. Roltgen $\mathrm{K}$ et al., Defining the features and duration of antibody responses to SARS-CoV-2 infection associated with disease severity and outcome. Sci Immunol. 2020; https://doi.org/10.1126/sciimmunol.abe0240.

\section{Table}

Table 1 The level of induced humoral immune responses after COVID-19 vaccination in percentages. 


\begin{tabular}{|c|c|c|c|c|c|c|c|}
\hline Vaccination Regimen / Level of Ab titer $(\mathrm{BAU} / \mathrm{ml})$ & $>10000$ & $>2500>10000$ & $>400<2500$ & $>200<400$ & $>50<200$ & $>10<50$ & $<10$ \\
\hline Convalescent non vaccinated (101) & $0 \%$ & $1 \%$ & $1 \% 6$ & $10,90 \%$ & $42,60 \%$ & $34,70 \%$ & $10,00 \%$ \\
\hline One shot of BNT162b 2 (12) & $0 \%$ & $0 \%$ & $0 \%$ & $16,70 \%$ & $25,00 \%$ & $41,70 \%$ & $16,70 \%$ \\
\hline Two shots of BNT162b 2 (11) & $0 \%$ & $0 \%$ & $45,50 \%$ & $18,20 \%$ & $36,40 \%$ & $0,00 \%$ & $0 \%$ \\
\hline CovD-19+/one shot of BNT 162 b2 (31) & $16,10 \%$ & $61,30 \%$ & $13,00 \%$ & $6,50 \%$ & $0,00 \%$ & $3,00 \%$ & $0 \%$ \\
\hline Covid-19+/two shots of BNT $162 \mathrm{~b} 2$ (6) & $16,70 \%$ & $16,70 \%$ & $16,70 \%$ & $0,00 \%$ & $50,00 \%$ & $0,00 \%$ & $0 \%$ \\
\hline CovD-19+/one shot of mRNA-1273 (5) & & $20,00 \%$ & $80,00 \%$ & $0,00 \%$ & $0,00 \%$ & $0,00 \%$ & $0 \%$ \\
\hline One shot of inactivated virus vaccine (6) & $0 \%$ & O\$6 & $0 \%$ & $16,70 \%$ & $66,70 \%$ & $0,00 \%$ & $16,70 \%$ \\
\hline Two shots of inactivated virus vaccine (12) & $0 \%$ & $0 \%$ & $0 \%$ & $0,00 \%$ & $41,70 \%$ & $41,70 \%$ & $16,70 \%$ \\
\hline COVD-19+/one shot of inactivated virus vaccine (5) & $0,00 \%$ & $0,00 \%$ & $40,00 \%$ & $40,00 \%$ & $20,00 \%$ & $0,00 \%$ & $0 \%$ \\
\hline CovD-19+/two shots of inactivated virus vaccine (8) & $0,00 \%$ & $0,00 \%$ & $50,00 \%$ & $37,50 \%$ & $0,00 \%$ & $12,50 \%$ & $0 \%$ \\
\hline One shot of Vaxzevria (1) & $0 \%$ & $0 \%$ & $0 \%$ & $0,00 \%$ & $100,00 \%$ & $0,00 \%$ & $0,00 \%$ \\
\hline Two shots of Vaxzevria (2) & $0 \%$ & $0 \%$ & $100,00 \%$ & $0,00 \%$ & $0,00 \%$ & $0,00 \%$ & $0 \%$ \\
\hline CovD-19+/one shot of Vaxzevria (4) & $0,00 \%$ & $50,00 \%$ & $50,00 \%$ & $0,00 \%$ & $0,00 \%$ & $0,00 \%$ & $0 \%$ \\
\hline CovD-19+/two shots of Vaxzevria (1) & $0,00 \%$ & $0,00 \%$ & $100,00 \%$ & $0,00 \%$ & $0,00 \%$ & $0,00 \%$ & $0 \%$ \\
\hline One shot of JNJ-78436735 (3) & $0 \%$ & $0 \%$ & $0 \%$ & $0,00 \%$ & $33,30 \%$ & $0,00 \%$ & $66,70 \%$ \\
\hline One shot of Sputnik V(1) & $0 \%$ & $0 \%$ & $0 \%$ & $0,00 \%$ & $0,00 \%$ & $100,00 \%$ & $0,00 \%$ \\
\hline Two shots of Sputnik V(5) & $0 \%$ & $0 \%$ & $20,00 \%$ & $0,00 \%$ & $60,00 \%$ & $20,00 \%$ & $0 \%$ \\
\hline CovD-19+/one shot of Sputnik V (2) & $0,00 \%$ & $50,00 \%$ & $0,00 \%$ & $50,00 \%$ & $0,00 \%$ & $0,00 \%$ & $0 \%$ \\
\hline
\end{tabular}

Between parentheses is the number of vaccine recipients in each category.

\section{Figures}




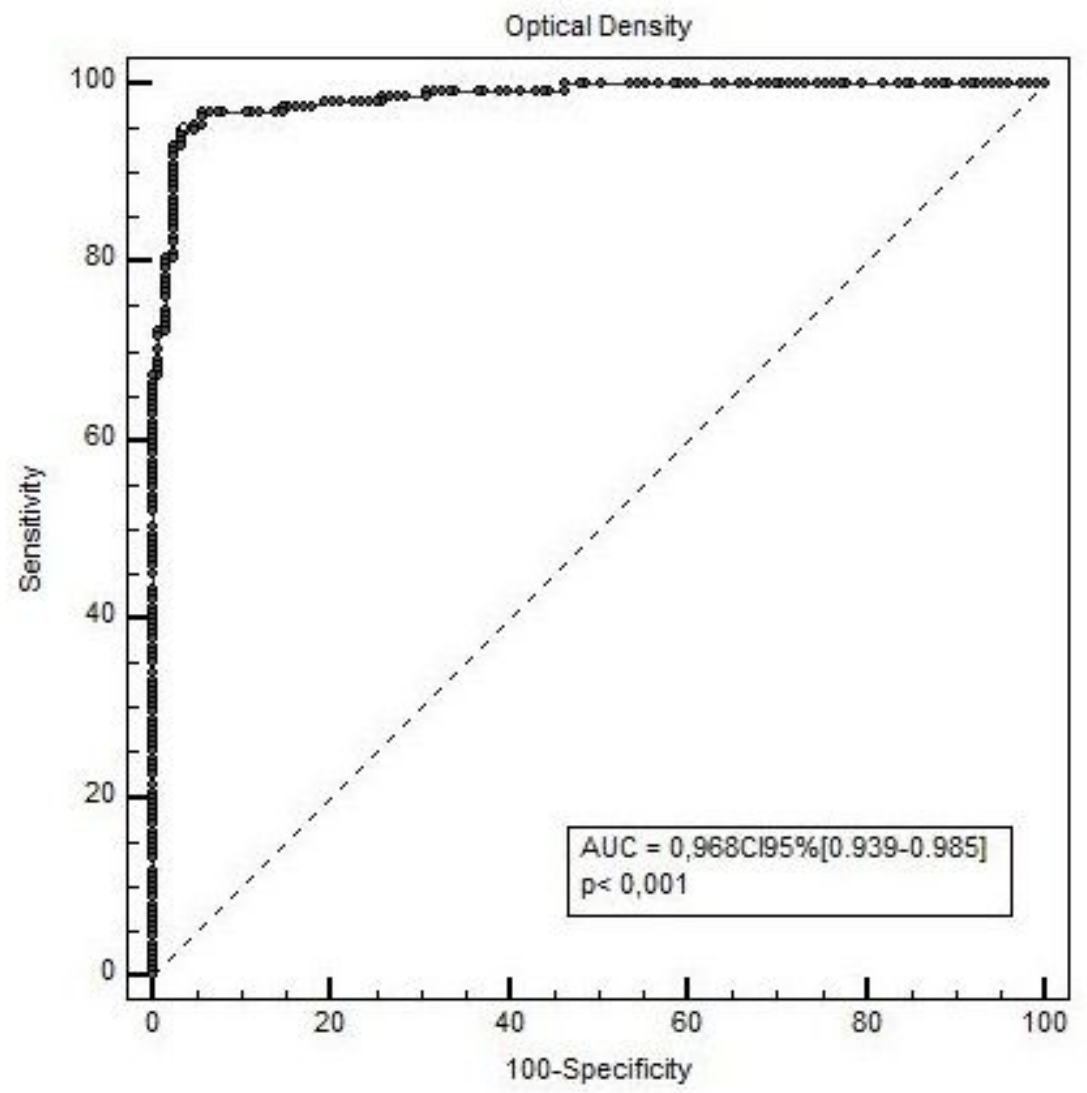

Figure 1

ROC curve for the in-house ELISA using optical density values. 


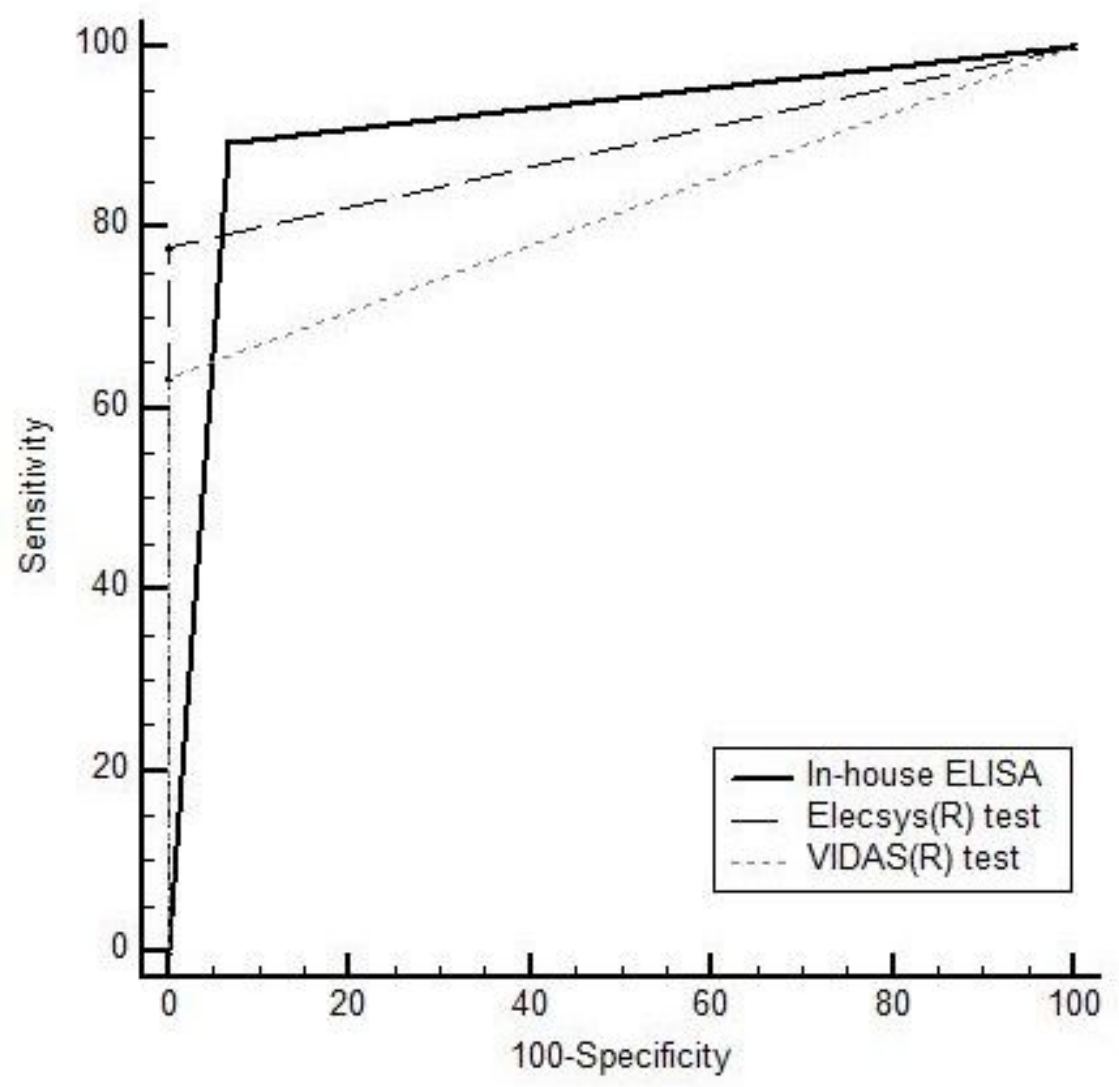

Figure 2

Comparison between the in-house Elisa qualitative results and those of the automated tests Vidas ${ }^{\circledR}$ SARS-CoV-2 IgG anti-RBD Biomérieux ${ }^{\circledR}$ and Elecsys ${ }^{\circledR}$ Anti-Nucleocapsid of SARS-CoV-2 Roche ${ }^{\circledR}$. 


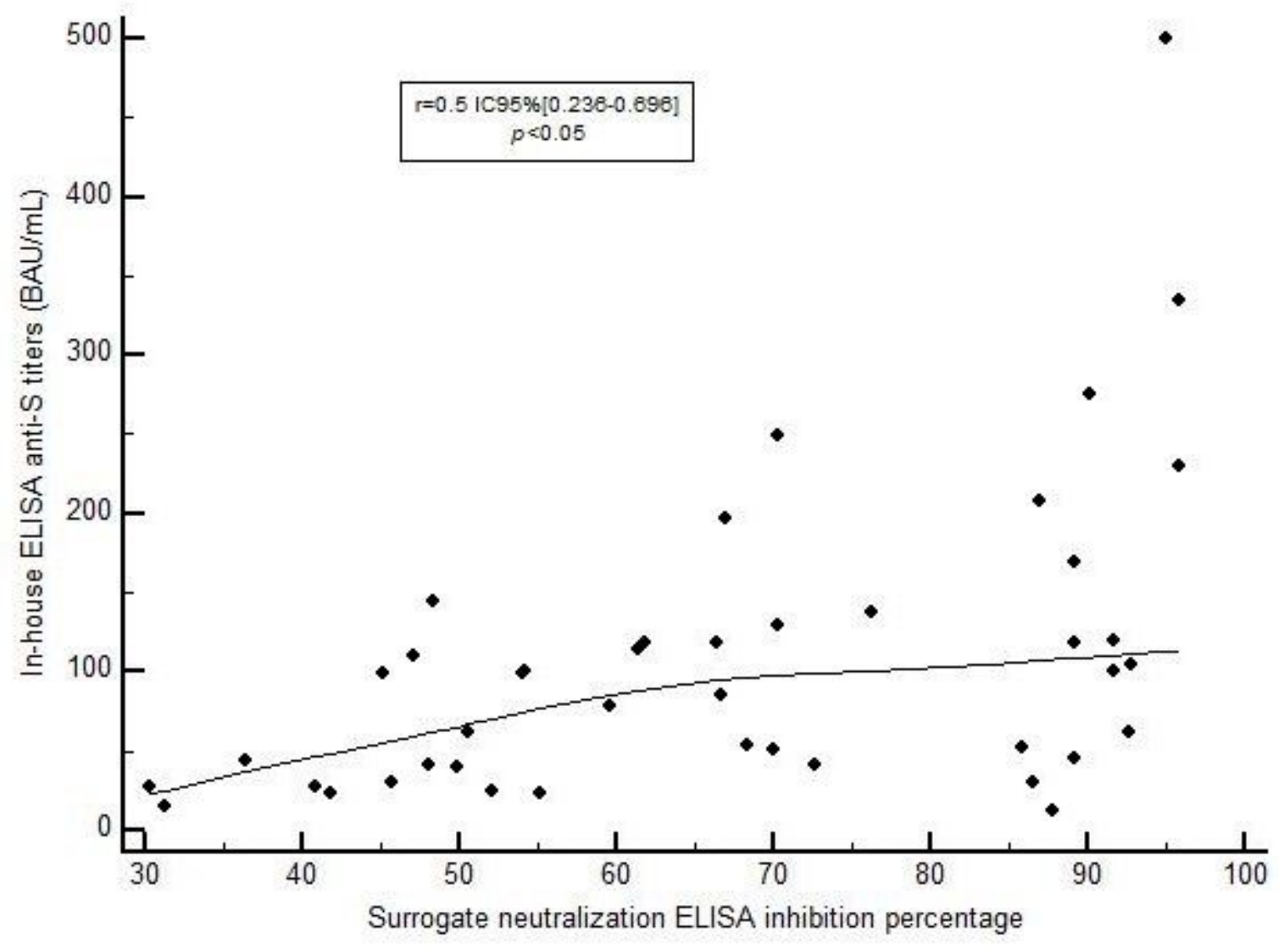

Figure 3

Correlation between the antibody titers $(\mathrm{BAU} / \mathrm{ml})$ of the in-house ELISA and the inhibition percentage by the surrogate neutralization ELISA (cPass ${ }^{\circledR}$ GenScript $\left.{ }^{\circledR}\right)$. 